\title{
Space VLBI Project VSOP
}

\author{
Makoto INOUE \\ Nobeyama Radio Observatory \\ Nobeyama, Minamisaku, Nagano 384-13, Japan
}

\begin{abstract}
A space VLBI satellite is planed to be launched in 1995 by the Institute of Space and Astronautical Science (ISAS), Japan. Together with this satellite, a worldwide collaborative project named the VLBI Space Observatory Program (VSOP) is under developed. Although the project is oriented to imaging mission for compact cores of active galactic nuclei and maser sources, it will open new aspect on all of VLBI applications. The basic design and parameters of VSOP project are reviewed.
\end{abstract}

\section{INTRODUCTION}

The VLBI Space Observatory Program (VSOP) was initiated as a cooperative project between the Institute of Space and Astronautical Science (ISAS) and the Nobeyama Radio Observatory (NRO), Japan, in the late 1980's. The VSOP satellite has an $8-\mathrm{m}$ deployable radio telescope and three radio astronomy receivers, and will be launched in 1995. It is expected to have a lifetime of more than three years. As the VSOP is a space VLBI imaging mission, the worldwide support of both link stations and ground observing telescopes is needed. Managed by the VSOP International Science Council (VISC), the Announcement of Opportunity (AO) will be released 1.5 years ahead of the launch of the VSOP satellite, and proposals will be screened by peer review. Some detailed descriptions are in the proceedings of the International VSOP Symposium (1992).

\section{ORBIT}

The orbit of the VSOP satellite is elongated (See Table 1) and precesses so that good UV coverage is expected over a wide area of the sky. As the orbit is not synchronous, about 6 hours per orbit, the UV coverage evolves day by day. Furthermore, since the apogee is not very high, VSOP is good for imaging rather than high resolution. There are, however, several constraints on the satellite. For instance, low declination sources may not have good UV coverage due to the avoidance angle of the Sun. The final situation depends on the exact time of the launch. 
Table 1. Orbit Parameters

\begin{tabular}{lr}
\hline Apogee Height & $20,000 \mathrm{~km}$ \\
Perigee Height & $1,000 \mathrm{~km}$ \\
Period & $\approx 6 \mathrm{hrs}$ \\
Inclination & $31^{\circ}$ \\
\hline
\end{tabular}

\section{RADIO TELESCOPE}

The main reflector is hexagonal in shape. Its surface is shaped by a tension truss structure of cable nets, and six extendible masts which tension the surface. Figure 1 shows an artist's impression of the VSOP satellite orbiting around the Earth. The accuracy of the mesh surface is $0.5 \mathrm{~mm}$ rms. The aperture is $50 \mathrm{~m}^{2}$, which is almost equal to the aperture of $8-\mathrm{m}$ diameter paraboloid, and the aperture efficiency is $40 \%, 56 \%$, and $44 \%$ at $1.6,5$, and $22 \mathrm{GHz}$, respectively.

Unlike radio telescopes on the ground, slew rate of the telescope is not so high. In addition, UV track rotates quickly, and hence the high speed switching method used in the Astrometric VLBI will not be applicable.

\section{RECEIVERS}

The VSOP satellite has three receivers at $1.6,5$, and $22 \mathrm{GHz}$ with uncooled frontends. Table 2 summarizes the receiver and system temperatures for each frequency, together with frequency coverage. Two IF channels are available so that simultaneous observations in any two of the three frequency bands, or at two frequencies within any one band, can be made. However, only left-hand circular polarization is available. Two synthesizers will be on board, and the observing frequencies can be set independently for the two channels in $1 \mathrm{MHz}$ steps. Two IF bandwidths $(16 \mathrm{MHz}$ and $32 \mathrm{MHz}$ ) are selectable (See Table 3 ). The sensitivity $(5 \Delta \mathrm{S})$ with a VLBA telescope at $5 \mathrm{GHz}$ (Tsys $=230 \mathrm{Jy}$ ) is, for example, $190 \mathrm{mJy}$ with $32 \mathrm{MHz}$ bandwidth and 2 minutes integration.

Table 2. Receiver and System Temperatures

\begin{tabular}{lccc}
\hline Band & $1.6 \mathrm{GHz}$ & $5 \mathrm{GHz}$ & $22 \mathrm{GHz}$ \\
\hline Frequency & $1.60-1.73 \mathrm{GHz}$ & $4.70-5.00 \mathrm{GHz}$ & $21.9-22.3 \mathrm{GHz}$ \\
TRX & $30 \mathrm{~K}$ & $50 \mathrm{~K}$ & $150 \mathrm{~K}$ \\
Tsys & $100 \mathrm{~K}$ & $120 \mathrm{~K}$ & $200 \mathrm{~K}$ \\
Tsys & $14,000 \mathrm{Jy}$ & $12,000 \mathrm{Jy}$ & $25,000 \mathrm{Jy}$ \\
\hline
\end{tabular}

\section{LINK AND OBSERVING MODE}

The received signal is sampled and sent to the link stations at $128 \mathrm{Mbps}$ at 15 $\mathrm{GHz}$. Each link station also sends a frequency reference signal from its hydrogen maser. A round-trip phase link signal is used to measure the error of the phase transfer. As the uplink and downlink frequencies are high and are very close to each other, the phase fluctuations caused by ionospheric disturbances are greatly reduced.

As a recorder is not installed on board, link stations will have VLBI terminals to record the VSOP satellite data. When no link station is accessible from the satellite, we cannot make any observations with the VSOP satellite. Consequently, a number of link stations, which are efficiently distributed around the world and ground observing telescopes, are needed to get good UV coverage. Five link stations are now planned at Usuda in Japan, Goldstone and Green Bank 
in the US, Canberra in Australia, and Madrid in Spain. ISAS runs the Usuda station, and NRAO operates Green Bank. The remainder are part of the JPL Deep Space Network.

VSOP observations will have three data parameters: the number of sampling bits, the number of IF channels, and their bandwidths. However, these are not mutually independent and should be chosen so that the total bit rate is 128 Mbps. In Table 3, the available combinations are listed.

Table 3. Observing Modes

\begin{tabular}{ccc}
\hline Bandwidth & bit & Channel \\
\hline $16 \mathrm{MHz}$ & 2 & 2 \\
$32 \mathrm{MHz}$ & 1 & 2 \\
$32 \mathrm{MHz}$ & 2 & 1 \\
\hline
\end{tabular}

\section{RECORDERS AND CORRELATORS}

At Usuda link station, a $\mathrm{K} 4$ recording terminal is installed which is capable of recording the 32-MHz modes; VLBA recorders will be installed in the other stations. The K4 tapes will be correlated with the VSOP correlator which is planned to be built in Japan. This correlator is the first one that is designed to cover items special to space VLBI, in particular, having a very wide fringe search window. At the VSOP correlator facility, data translation systems from VLBA to $\mathrm{K} 4$, and from $\mathrm{S} 2$ to $\mathrm{K} 4$ will be installed, to handle all types of recording systems. The S2 system has been developed in Canada and is used in Australia. VLBA tapes will also be correlated using the VLBA correlator.

\section{References}

Frontiers of VLBI, 1992, Proceedings of the International VSOP Symposium and of the mm-VLBI Workshop, eds. H. Hirabayashi, M. Inoue and $\mathrm{H}$. Kobayashi, Universal Academy Press, Inc., Hongo, Tokyo, Japan. 


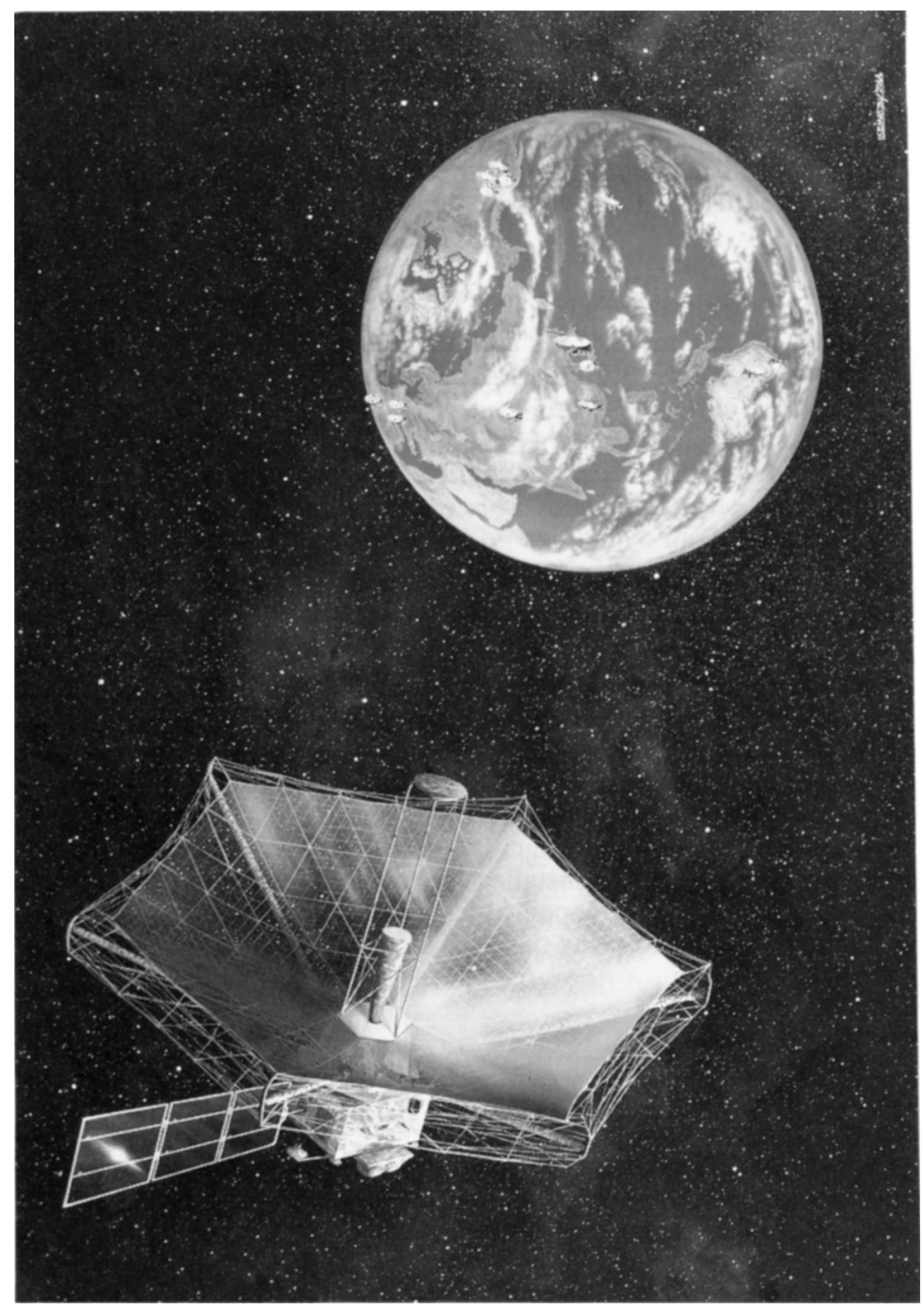

Figure 1. An artist's impression of the VSOP satellite orbiting the Earth. 